\title{
Investigation of Residual Stress in Laser Formed Mild Steel Plates Using Neutron Diffraction
}

\author{
Stefan Knupfer ${ }^{1}$, Anna M. Paradowska ${ }^{2}$, Oliver Kirstein ${ }^{3}$ and \\ Andrew Moore ${ }^{1, a}$ \\ ${ }^{1}$ Heriot-Watt University, School of Engineering \& Physical Sciences, Edinburgh, UK \\ ${ }^{2}$ ISIS Neutron Scattering Facility, Rutherford Appleton Laboratory, Didcot, UK \\ ${ }^{3}$ Australian Nuclear Science and Technology Organisation, NSW AUSTRALIA \\ aa.moore@hw.ac.uk
}

Keywords: Laser forming, neutron diffraction, residual stress

\begin{abstract}
Forming of metal plates with a high-power laser beam is a flexible materials forming technique. Bending results from the establishment of a steep temperature gradient through the material thickness which leads to non-uniform thermal expansion/contraction and subsequently residual stresses. It is important to characterize these residual stresses as a function of process parameters such as line energy (LE) to optimize treatment conditions and to gain an insight into the mechanism of the formation of the final geometries.

Non-destructive neutron diffraction measurements were carried out on ENGIN-X at ISIS and KOWARI at ANSTO to map the residual stress distribution around the heat affected zone (HAZ) of laser deformed mild steel plates for single and multiple passes as a function of line energy (LE), the primary laser forming process parameter. It was found that in the centre of the HAZ, longitudinal residual stresses are tensile and dominant, transverse stresses are predominantly tensile and normal stresses are compressive and close to zero. The residual strain in longitudinal direction increased with LE and number of passes until yielding. Even higher heat input decreased the magnitude of the cusp, but not its total height. The comparison of a stress-free reference sample, measured at both facilities, showed a small discrepancy in the lattice spacing corresponding to $\sim 85 \mu$ strain which is insignificant with respect to the experimental values measured.
\end{abstract}

\section{Introduction}

Laser forming is significantly different from other hot working processes with its very fast heating and cooling cycles. During the laser forming process the surface of the material is heated by a defocused laser beam, which traverses the work piece and creates a steep thermal gradient through the thickness of the sample. The thermal expansion of the surface introduces thermal stress including plastic strains due to the constraint of the surrounding cold material, which result in bending and/or shortening the material (temperature gradient mechanism) or produce a local buckling (buckling mechanism) [1,2]. Therefore the forming of the sheet material arises from the setting up of complex residual stress (RS) fields associated with the thermal gradient, induced by the laser heating.

Some studies have already been carried out to map the residual stress after laser forming around the heat affected zone (HAZ) [3,4]. These studies showed that directly below the laser scan path longitudinal strains are tensile and dominant, normal strains are compressive and transverse strains slightly tensile. Their magnitude decreased with number of laser scans applied and a strong relationship between the distribution of residual elastic strains and microstructure was found. However, the study was performed for specimens formed at a single laser power only.

The characterization of residual stresses around the HAZ as a function of line energy (LE), which is defined as laser power divided by the moving speed of the laser beam, is of special interest in the 
iterative approach to laser forming where the sample converges in multiple laser scan passes towards the desired shape. The process has proven its potential in the past, either as a tool of post process distortion control or rapid prototyping in form of forming $3 \mathrm{D}$ shapes, such as pillow and saddle [5,6]. Therefore, if laser forming is to be used in an industrial application in the future, it is extremely important to fully understand its effect not only on microstructure and mechanical properties, but also on the residual stress distribution after laser forming, because it has important consequences on the performance of engineering components.

Therefore, this study aims to characterize these residual stresses as a function of process parameters such as line energy in order to optimize the treatment conditions for laser forming in low carbon steel (AISI1010). Neutron diffraction at two different facilities was used to perform strain scans across the HAZ on samples, treated in a LE-range between $40-130 \mathrm{~J} / \mathrm{mm}$ with one and three laser scan passes. The RS measurement was carried out specifically as part of a detailed study to define process parameters for our approach to iterative laser forming $[5,6]$.

\section{Experimental details}

The material investigated in this study was low carbon steel (AISI1010) [7]. Square test specimens of AISI1010 of dimension $80 \times 80 \times 2.8 \mathrm{~mm}^{3}$ were laser cut from the parent sheet. The plates were not annealed because the RS measured in the non-formed parent material was negligible compared to that induced by laser forming. The forming process was applied by either one or three laser scan passes along the centreline, as shown in Figure 1. The location of the laser scan pass stayed the same for all the samples which were scanned 3 times. Between subsequent passes, 60 sec. were allowed for the sample to cool down, minimising any increase in peak temperature of the material with each pass. Sample conditions, induced distortion and measurement facility for the residual stress are shown in Table 1.

Table 1: Sample conditions

\begin{tabular}{|c|c|c|c|}
\hline $\begin{array}{c}\text { No. of laser scan } \\
\text { passes }\end{array}$ & $\begin{array}{c}\text { Line Energy } \\
{[\mathbf{J} / \mathbf{m m}]}\end{array}$ & $\begin{array}{c}\text { Final bend angle } \\
{\left[{ }^{\circ}\right]}\end{array}$ & $\begin{array}{c}\text { Measurement } \\
\text { facility }\end{array}$ \\
\hline 1 & 40 & 0.66 & ANSTO \\
\hline 1 & 70 & 0.72 & ISIS \\
\hline 1 & 100 & 0.89 & ISIS \\
\hline 1 & 130 & 0.88 & ISIS \\
\hline 3 & 40 & 1.00 & ANSTO \\
\hline 3 & 70 & 1.88 & ANSTO \\
\hline 3 & 100 & 2.61 & ISIS \\
\hline 3 & 130 & 2.79 & ANSTO \\
\hline
\end{tabular}

A TRUMPF TLF 2700 Turbo $\mathrm{CO}_{2}$ laser with $2.7 \mathrm{~kW}$ in $\mathrm{CW}$ (continuous wave) mode was used for the experiments with the laser beam beyond its focus, providing a beam diameter of $9 \mathrm{~mm}$. To vary the heat input, the laser power was fixed at $600 \mathrm{~W}$ and the laser scan speed was adjusted to provide the desired LE (laser power divided by the moving speed of the laser).

The bend angle was measured with a single point triangulation probe connected to a National Instrument data acquisition card (NI USB-6221 M series). During a shape scan the probe spot was scanned across the HAZ in the "transverse" direction (Figure 1). The final bend angle, shown in Table 1, is the average of 15 separate measurements made at $5 \mathrm{~mm}$ spacing in the "longitudinal direction" along the laser scan path. In order to enhance the absorption of the laser light, samples were cleaned with acetone and hand sprayed with graphite prior to the laser treatment. 


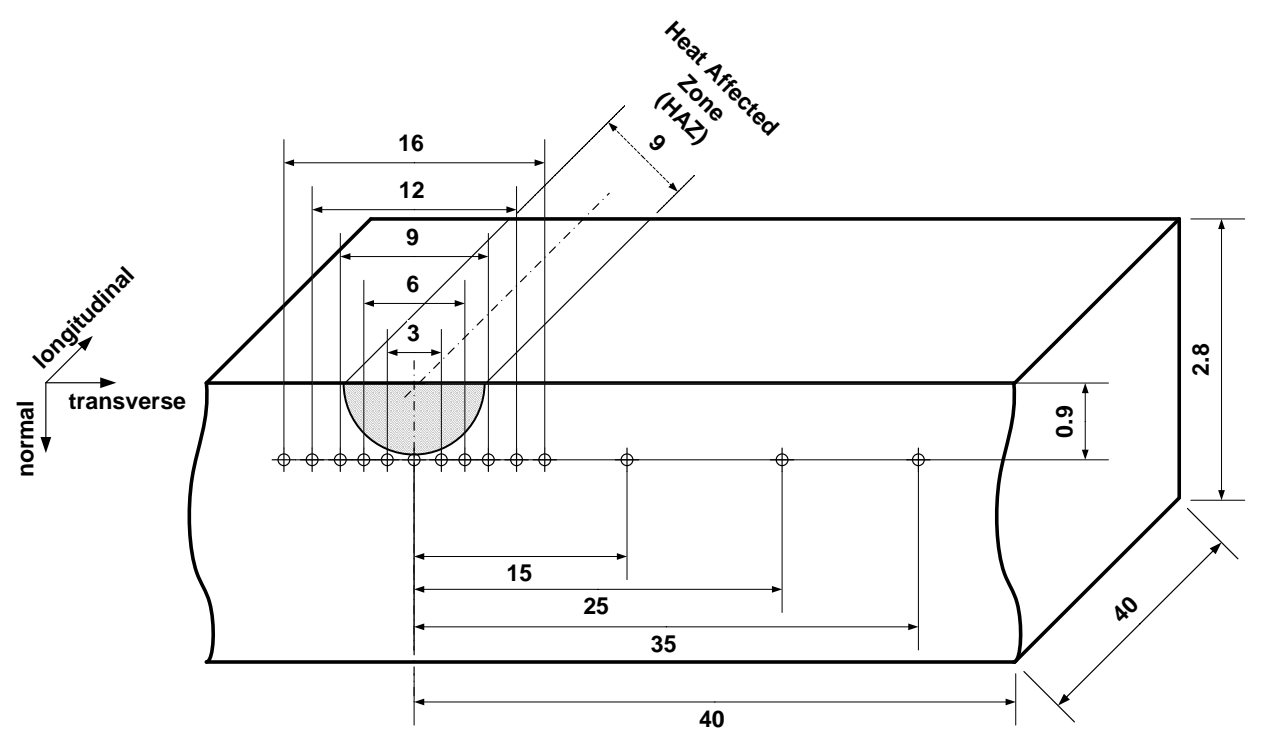

Figure 1: RS-measurement points across the HAZ

RS measurements were made at two neutron facilities using two different strain scanning modes, namely monochromatic (constant wavelength) on the KOWARI strain scanner at the Australian research reactor OPAL and time-of-flight on the ENGIN-X neutron scattering diffractometer at ISIS, Rutherford Appleton Laboratory, UK.

The strain measurements on KOWARI were done using the $\mathrm{Fe}(211) \mathrm{Bragg}$ reflection observed at a scattering angle $2 \theta$ of $\sim 90^{\circ}$ with a monochromatic thermal neutron beam of $1.66 \AA$, which was extracted from a double focusing Si (400) monochromator at a take-off angle of $\sim 75^{\circ}$. A gauge volume of $0.5 \times 0.5 \times 15 \mathrm{~mm}^{3}$ was used to measure the transverse direction across the HAZ, for the normal and longitudinal directions a gauge volume of $1 \times 1 \times 1 \mathrm{~mm}^{3}$ was chosen. Accurate sample alignment and surface finding was performed on each sample separately using the neutron beam.

ENGIN-X is the dedicated time-of-flight engineering diffractometer at the ISIS pulsed neutron source. The instrument has two detector banks centred on horizontal scattering angles of $\pm 90^{\circ}$, enabling simultaneous measurement of two orthogonal strain components. Measurements were made following the standard guidelines [8,9]. As ENGIN-X utilises a spallation neutron source, it is a time-of-flight facility and multiple diffraction peaks were acquired simultaneously. This enables lattice spacings to be obtained directly using a Pawley-Rietveld refinement (GSAS), after time focussing the raw data from each detector bank. Accurate alignment of the thin specimens was achieved using the software SScanSS. A gauge volume of $1 \times 1 \times 1 \mathrm{~mm}^{3}$ was used, which allowed the measurements to be made in an acceptable time.

For measurements at both facilities, the specimens were positioned to permit determination of stresses in the three primary working orthogonal directions of the deformed plates (Figure 1). Stress-free $d_{0}$ measurements were performed on a small cube sample $\left(2 \times 2 \times 2.8 \mathrm{~mm}^{3}\right)$, carefully cut from both the parent material and HAZ. For the measurement itself, 4 of those cubes were glued together.

\section{Results}

The experimental results of both measurements are presented in the following.

Table 2 shows a comparison of the $d$-spacing in in-plane and normal direction of a cube sample, which was cut from a "raw" plate (no heat input) and was measured at both facilities.

Table 3 compares the stress-free $d_{0}$ cut from the HAZ of samples, scanned with the highest and lowest LE, considered in this study.

The negligible discrepancy of a back-to-back measurement from the $d_{0}$ reference sample carried out at both facilities justifies plotting results of residual strain measurements, made at the different institutes, together in the same graph, e.g. Figure 3. 
Table 2: Comparison of $d$-spacing of "stress-free" (no heat input, LE $=0 \mathrm{~J} / \mathrm{mm}$ ) cubes, measured at both facilities, ANSTO and ISIS

\begin{tabular}{|c|c|c||c|c|}
\hline$d$-spacing $[\AA]$ & in-plane & Error & Normal & error \\
\hline ANSTO & 1.1706 & $1.4388 \mathrm{E}-05$ & 1.1705 & $6.5091 \mathrm{E}-07$ \\
\hline ISIS & 1.1705 & $6 \mathrm{E}-05$ & 1.1705 & $5 \mathrm{E}-05$ \\
\hline
\end{tabular}

Table 3: Comparison of $d$-spacing at different LE (measured at ANSTO)

\begin{tabular}{|c||c|c||c|c|}
\hline \multirow{2}{*}{$\mathrm{LE}[\mathrm{J} / \mathrm{mm}]$} & \multicolumn{4}{|c|}{$d$-spacing $[\AA]$} \\
\cline { 2 - 5 } & in-plane & error & normal & error \\
\hline 0 & 1.1706 & $1.4388 \mathrm{E}-05$ & 1.1705 & $6.5091 \mathrm{E}-07$ \\
\hline 40 & 1.1706 & $4.9507 \mathrm{E}-06$ & 1.1706 & $6.5071 \mathrm{E}-06$ \\
\hline 130 & 1.1705 & $7.3798 \mathrm{E}-06$ & 1.1705 & $9.1886 \mathrm{E}-06$ \\
\hline
\end{tabular}

The graphs in Figure 2 show an example of residual strain and RS in all three perpendicular directions across the HAZ, measured at a sample scanned once at $\mathrm{LE}=70 \mathrm{~J} / \mathrm{mm}$. Lattice spacing was converted to residual strains and stresses using the standard three dimensional Hooke's law as shown in equation 1 [10]. The bulk elastic modulus $E$ for AISI1010 was 190GPa with Poisson's ratio of $v=0.3[7]$.

$$
\sigma_{x x}=\frac{E}{1+v} \varepsilon_{x x}+\frac{E v}{(1+v)(1-2 v)}\left(\varepsilon_{x x}+\varepsilon_{y y}+\varepsilon_{z z}\right)
$$

It can be seen that in the centre of the HAZ residual stresses and strains in longitudinal direction are tensile and dominant and the normal ones are compressive. In the transverse direction, residual strains are compressive and residual stresses are close to zero or slightly tensile. Outside the laser beam footprint the RS in all three perpendicular directions is close to zero.

a)

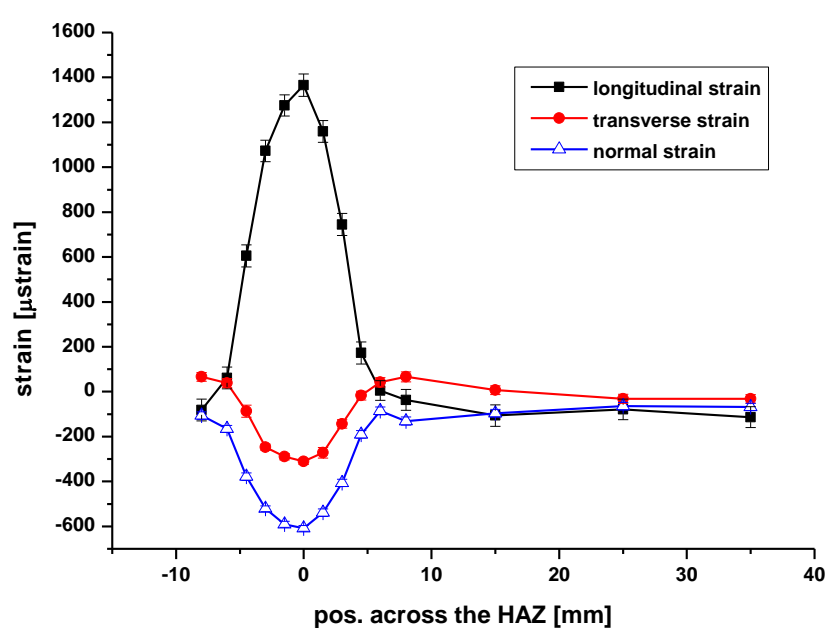

b)

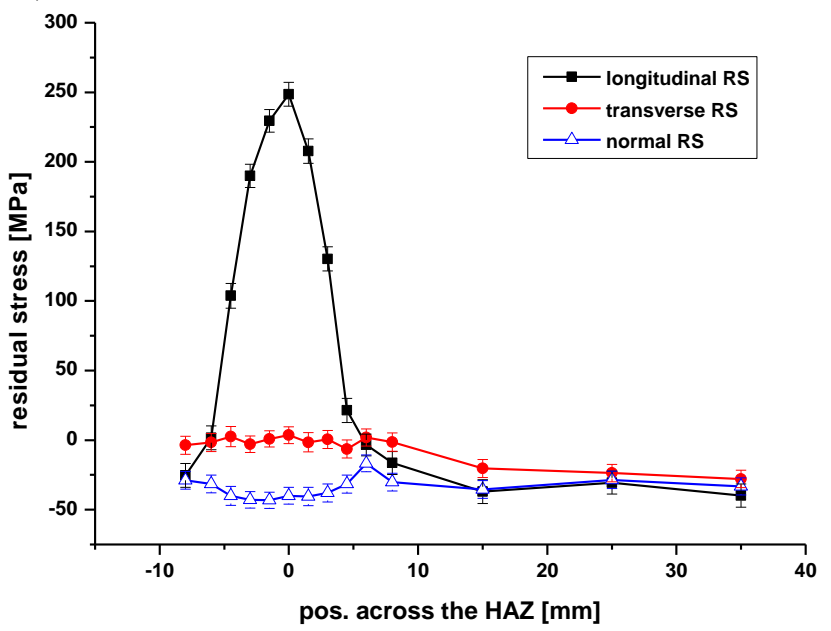

Figure 2: (a) Residual strain across the HAZ and (b) residual stress across the HAZ; LE $=70 \mathrm{~J} / \mathrm{mm}$, 1 pass

Figure 3 shows the evolution of the dominant longitudinal residual strain across the HAZ with increasing LE at one and three passes. It can be seen that with increasing heat input, associated with higher LE and/or multiple passes, the longitudinal residual strain increases until a threshold is reached. This threshold is due to the material yielding in tension and is discussed in the next section. 
a)

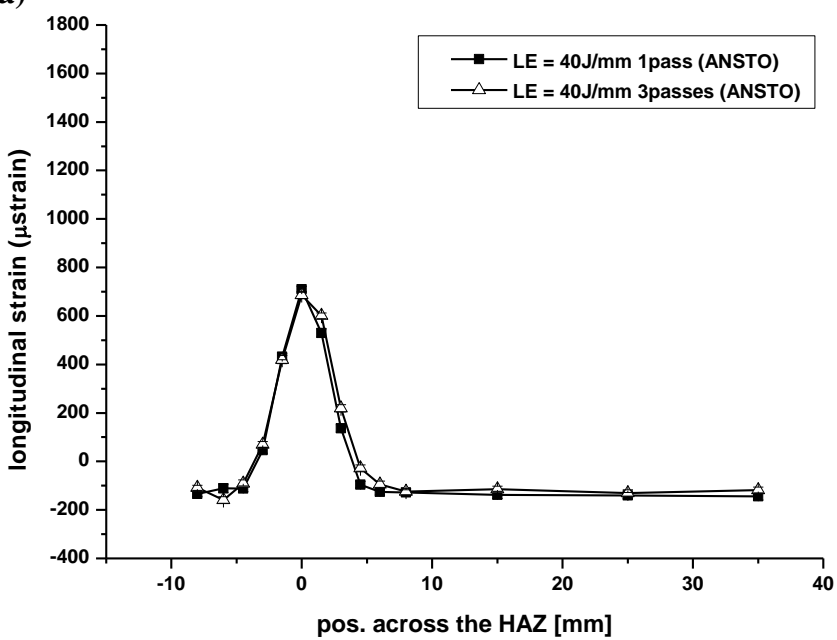

c)

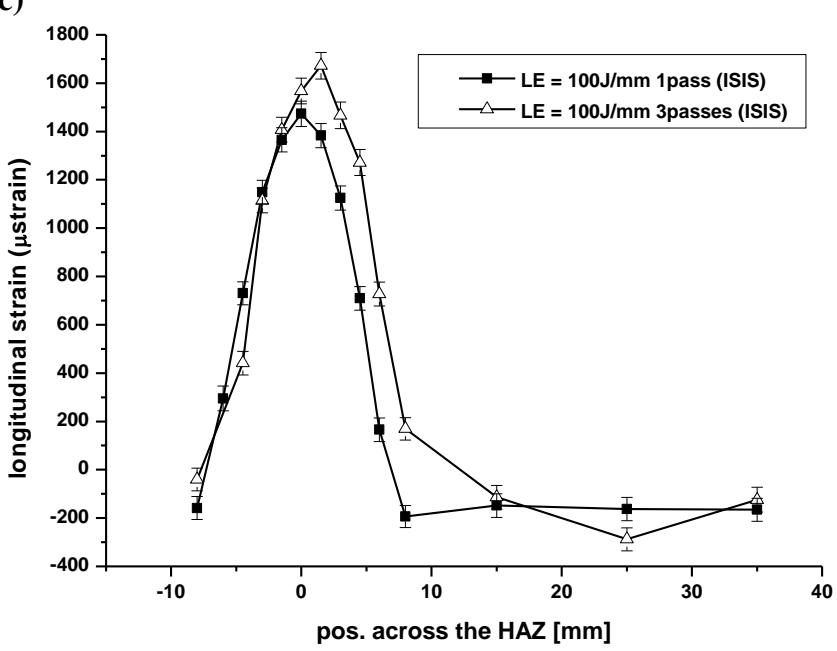

b)

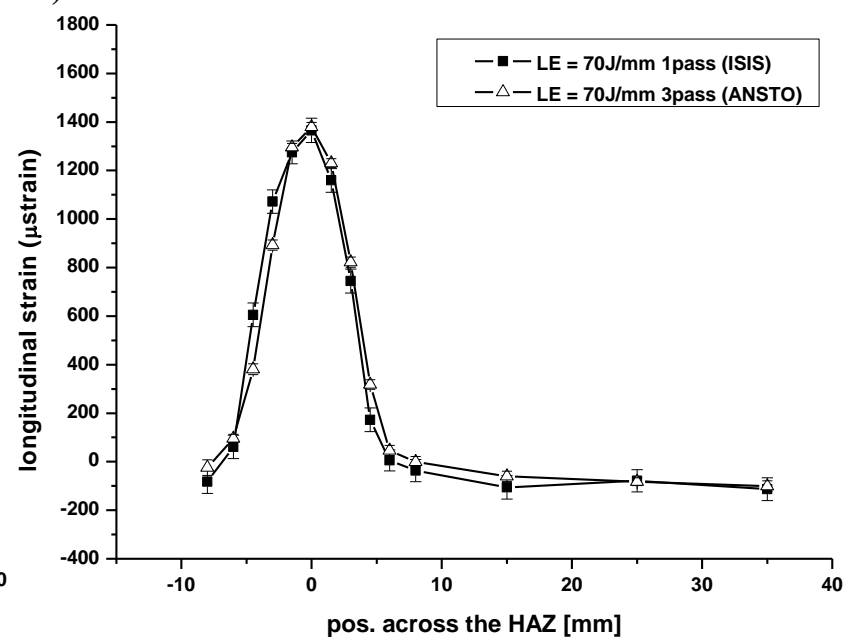

d)

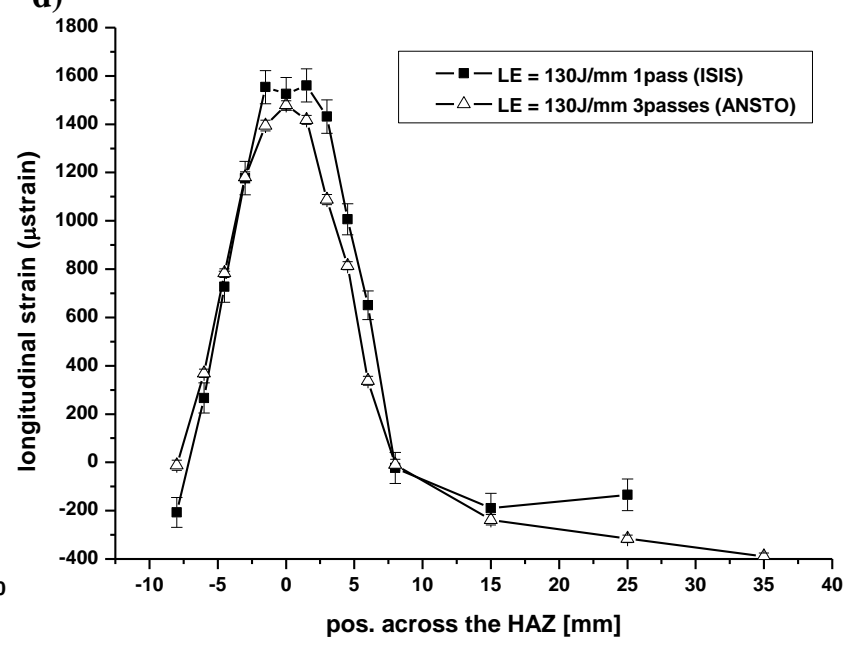

Figure 3: Longitudinal strain across the HAZ (1 and 3passes): (a) LE $=40 \mathrm{~J} / \mathrm{mm}$; (b) $\mathrm{LE}=70 \mathrm{~J} / \mathrm{mm}$; (c) $\mathrm{LE}=100 \mathrm{~J} / \mathrm{mm}$; (d) $\mathrm{LE}=130 \mathrm{~J} / \mathrm{mm}$

\section{Discussion}

Stress-free $d_{0}$ cubes carefully cut from the HAZ of samples, scanned at LE $=40 \mathrm{~J} / \mathrm{mm}$ and LE $=$ $130 \mathrm{~J} / \mathrm{mm}$, which is the lowest and highest LE investigated in this study respectively (Table 3), showed only a minor discrepancy in lattice plane spacing, due to the heat of the laser beam. It is equivalent to $\sim 85 \mu$ strain, which is an order of magnitude smaller than the strain measured in the bulk samples.

From Figure 2b, which maps the RS across the HAZ of a sample, scanned once at LE $=70 \mathrm{~J} / \mathrm{mm}$, it can be seen that in the centre of the HAZ longitudinal stresses are tensile and dominant, transverse stresses are predominantly tensile and normal stresses are compressive and close to zero. The residual strain peak is most significantly in the longitudinal direction, because the shrinkage as a consequence of inhomogeneous temperature distribution is impeded to a much larger extent than, for example, in the transverse direction. Therefore, with increasing heat input, associated with higher LE and/or multiple passes, the longitudinal residual strain increases until a threshold is reached, due to the material yielding in tension, as shown in Figure 3a-c. For samples scanned at LE $=130 \mathrm{~J} / \mathrm{mm}$ (Figure 3d), the magnitude of the strain cusp decreased, but its total height was found to remain constant, as shown in Figure 4a. A similar behaviour was observed for the full width half maximum (FWHM) of the peak (Figure $4 \mathrm{~b}$ ), which is limited due to the laser beam diameter.

It was also found in a mechanical and metallurgical study, to be published elsewhere, that with higher heat input, yield strength, ultimate tensile strength and hardness in the laser formed HAZ increases. Furthermore, the microstructure becomes visibly affected in the form of a bainiticmartensitic microstructure in the HAZ and with further depth, small recrystallized grains and carbon dissolution at the grain boundaries are most likely to be observed. However, these 
characteristics were found for samples scanned at $\mathrm{LE}=130 \mathrm{~J} / \mathrm{mm}$, (Figure 3d), whilst those at lower LE's and at multiple passes had no adverse affect.

a)

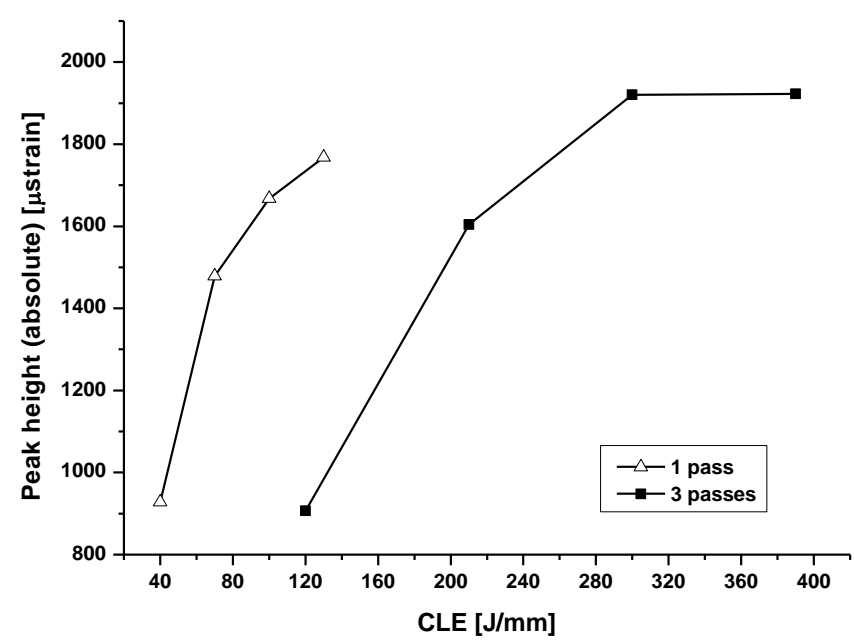

b)

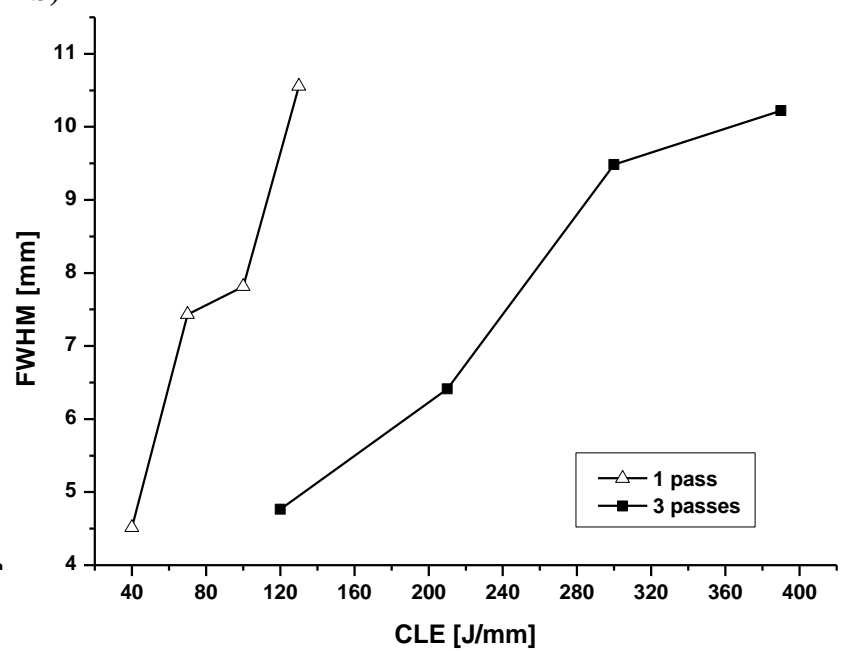

Figure 4: Analysis of the longitudinal strain peak with heat input: (a) peak height and (b) FWHM; parameter "CLE" is "cumulative LE" (LE times number of laser scan passes)

\section{Conclusion}

The effect of increasing heat input in laser forming, either in form of higher LE or multiple laser scan passes, on the RS was shown by an analysis of the dominant longitudinal residual strain peak, whose overall height and FWHM was found to show threshold behaviour due to the onset of yielding in tension. $d_{0^{-}}$cubes cut from the HAZ of samples, treated at different LE had no observable variation in lattice spacing and provided accurate stress-free reference for the measurement carried out at two different neutron facilities.

Regarding the iterative approach to laser forming and its potential industrial application, the RS distribution has to be taken into account, because large RS gradients change the stress intensity inside a sample and superimpose with in-service stresses. However, as shown in this study, the amount of the RS could be controlled by adjusting the laser parameters accordingly.

\section{Acknowledgements}

This work was supported by the Engineering and Physical Sciences Research Council [grant number GR/S12395/01]. Andrew Moore acknowledges the support of AWE through its William Penney Fellowship scheme. Experiments at the ISIS Pulsed Neutron and Muon Source were supported by a beamtime allocation from the Science and Technology Facilities Council [grant numbers RB920519 and RB910542].

\section{References}

[1] F. Vollertsen, Proc.of LANE'04, Vol. 1, pp. 345-360

[2] H. Arnet, F. Vollertsen, 1995; Proc. Inst. Mech. Eng. 209, (1995)

[3] M. Topic, P. McGrath, W.J. Vorster, S.Y. Zhang, R. Bucher, A. Venter, A.M. Korunsky, J. Strain Anal. Eng., Vol. 42, p. 497-504 (2007)

[4] A.M. Venter, M.W. van der Watt, R.C.Wimpory, R. Schneider, P.J. McGrath, M. Topic, Mater. Sci. Forum, Vol. 571-572 (2008), p.63-68

[5] R. McBride, F. Bardin, M. Gross, D.P. Hand, J.D.C. Jones, A.J. Moore J Phys D, 38 4027-4036 (2005)

[6] S. Knupfer, S. Rouquette, A.J. Moore, in Proc. of the IWOTE'08, p. 205-215

[7] Metals Handbook $9^{\text {th }}$ Edition, Volume 1, "Properties and selection: irons and steels", ASME, Metals Park, Ohio 44073

[8] IS0/TTA3, Polycrystalline materials - Determination of residual stresses by neutron diffraction, in: Technology trends assess., ISO, 2001.

[9] D. C. I. T. 21432, Non-destructive testing: British Standards Institute, 2005.

[10] M. T. Hutchings, P. J. Withers, T. M. Holden, T. Lorentzen, CRC Press, Boca Raton, FL, USA, 2005, p. 424. 